\title{
Identification of differentially expressed genes, IncRNAs and miRNAs which are associated with tumor malignant phenotypes in hepatoblastoma patients
}

\author{
Sida Liu ${ }^{1, *}$, Fujing Xie ${ }^{2, *}$, Xiaohong Xiang ${ }^{3}$, Sinan Liu ${ }^{4,3}$, Shunbin Dong ${ }^{3}$, Kai Qu ${ }^{3}$ and \\ Ting Lin ${ }^{4,3}$ \\ ${ }^{1}$ Department of The Second General Surgery, Shaanxi Provincial People's Hospital, Xi'an 710068, China \\ ${ }^{2}$ Department of Pediatrics, Liaocheng People's Hospital, Taishan Medical College, Liaocheng 252000, China \\ ${ }^{3}$ Department of Hepatobiliary Surgery, The First Affiliated Hospital of Xi'an Jiaotong University, Xi'an 710061, China \\ ${ }^{4}$ Department of Surgical Intensive Care Units, The First Affiliated Hospital of Xi'an Jiaotong University, Xi'an 710061, China \\ *These authors have contributed equally to this work \\ Correspondence to: Kai Qu, email: joanne8601@163.com \\ Ting Lin, email:947119451@qq.com \\ Keywords: hepatoblastoma; IncRNA; miRNA; mRNA; malignant phenotype \\ Received: August 02, $2017 \quad$ Accepted: August 24, $2017 \quad$ Published: October 31, 2017 \\ Copyright: Liu et al. This is an open-access article distributed under the terms of the Creative Commons Attribution License 3.0 \\ (CC BY 3.0), which permits unrestricted use, distribution, and reproduction in any medium, provided the original author and source \\ are credited.
}

\section{ABSTRACT}

Hepatoblastoma (HB) is one of the most common hepatic malignancies in the pediatric population. HB are composed of a variety of tumors, which derived from different origins and had varying clinical outcomes. However, the unclear underlying mechanisms of HB limited exploring novel biomarkers and effective therapeutic targets. We searched microarray datasets on Gene Expression Omnibus (GEO) database and selected GSE75271 and GSE75283 datasets for comprehensive analysis. Weighted gene correlation network analysis (WGCNA) was employed to identify genes which were associated with tumor malignant phenotypes, including HB subtypes, Cairo classification and tumor stage. Coexpression analysis of identified genes was also performed and IncRNA-miRNA-mRNA network was finally conducted. Our results showed that a total of 22 IncRNAs, 13 miRNAs and 66 mRNAs were identified to be associated with tumor malignant phenotypes. Mechanistically, these molecules might promote the malignant phenotypes via regulating metabolic pathways. Among of them, 6 miRNAs (hsa-miR-106b, hsa-miR-130b, hsa-miR-19a, hsa-miR-19b, hsamiR-20a and hsa-miR-301a), 8 IncRNAs (NR_102317, XR_245338, XR_428373, XR_924945, XR_929728, XR_931611, XR_935074 and XR_946696), and 6 mRNAs (EGFR, GAREM, INSIG1, KRT81, SAR1B and SDC1) were selected to conduct a IncRNAmiRNA-mRNA network. Taken together, our findings provide evidence for exploring molecular mechanisms of HB. Those identified malignant phenotype-associated molecules might be potential biomarkers and anti-cancer therapeutic targets in future.

\section{INTRODUCTION}

Hepatoblastoma (HB) is the most common malignant liver tumor in children, accounting for approximately $50 \%$ of pediatric hepatic-related cancers [1]. The incidence of HB in children with age $<15$ is about one per million $(1 / 1,000,000)$, and nearly $20 \%$ of those patients already have a synchronous metastasis at the first diagnosis. Despite recent advances in treatment, such as surgical resection, adjuvant chemotherapy, and liver transplantation, the prognosis in advanced HB stages still remains poor $[2,3]$. It has been accepted that $\mathrm{HB}$ are 
composed of a variety of tumors deriving from different immature liver precursors, including hepatocytes, biliary, and other epithelial or mesenchymal cells, which caused significant tumor heterogeneity [4, 5]. For decades, researchers have observed the varying clinical outcomes in patients with different histological subgroups [5-9]. In addition, other parameters, such as tumor stage, distant metastasis, multifocality, patient age and birth weight, have also been reported to be associated with prognosis $[10,11]$. However, the underlying mechanisms remains unknown. A thorough understanding of molecular mechanisms regarding tumor progression is essential for exploring effective therapeutic targets against HB.

Studies have revealed that only a small proportion $(1 \%-2 \%)$ of the genome encodes proteins, and the majority of the mammalian genome encodes plenty of non-coding RNAs [12-14]. As an important member of the non-coding RNAs, microRNAs (miRNAs), referred to 18-25 nucleotides, have been clearly demonstrated to regulate a variety of cellular processes via recognizing the 3'-untranslated regions of specific mRNAs and suppressing the expression of target genes [15]. Long non-coding RNAs (lncRNAs), another sort of non-coding RNAs with more than 200 nucleotides, are found to play pivotal roles in chromatin remodeling, transcription regulation and posttranscriptional mRNA processing [16-19]. Recently, increasing studies reported that IncRNAs could also function as competing endogenous RNAs (ceRNAs) by competitively binding to miRNAs through their miRNA response elements (MRE) [20]. These ceRNAs usually share MRE with other coding transcripts and therefore act as sponges for that cluster of miRNAs, protecting the targeted mRNA transcripts from degradation [21]. Although the functional mechanism that lncRNAs act as ceRNAs leading to miRNA deregulation has been observed in multiple malignancies, the lncRNA-miRNA-mRNA network in $\mathrm{HB}$ is far from being fully investigated.

In the present study, we performed a comprehensive analysis based on mRNA, lncRNA and miRNA expression profiling data derived from $50 \mathrm{HB}$ patients and 5 controls, and identified differentially expressed mRNA, lncRNA and miRNA in HB. Next, we employed weighted gene correlation network analysis (WGCNA) and coexpression analysis to select the modules which are associated with tumor malignant phenotypes. Based on above results, we finally conducted several potential lncRNA-miRNAmRNA networks in $\mathrm{HB}$.

\section{RESULTS}

\section{Identification of the malignant phenotype- associated mRNAs and IncRNAs in HB}

GSE75271 was selected for identify differentially expressed mRNA and lncRNAs in HB patients [22]. As shown in Figure 1A, Then, we performed a probe level analysis of GSE75271 by linear models for microarray data (LIMMA). Those probes with $P$-value less than 0.05 and foldchange more than 2.0 were identified as differentially expressed probes. One hundred ninety-four probes were primary selected, which were all downregulated in HB patients (Figure 1B). Next, 194 probes were annotated by Affymetrix microarray annotation files according to previous reported method [23]. Finally, we identified a total of 61 lncRNAs and 133 mRNAs from 194 differentially expressed probes (Figure 1C).

To further explore the malignant phenotypeassociated mRNAs and lncRNAs, we performed a weighted gene correlation network analysis (WGCNA) (Figure 2A) and divided 194 differentially expressed probes into eight module eigengenes (ME), including MEgrey, MEred, MEblue, MEgreen, MEbrown, MEmagenta and MEblack (Figure 2B). Next, we employed module-trait relationship analysis to conduct the association between eight MEs and 13 clinical traits (including race, sex, age, HB subtypes, Cairo classification, tumor stage, CTNNB1, NFE2L2 and TERT mutation status, histological types, prognosis, tumor recurrent status and survival time). Interestingly, we found that one module (MEblue) was negatively associated with HB subtypes, Cairo classification and tumor stage, with $P$-values of $1 \times 10^{-7}, 2 \times 10^{-8}$ and $1 \times 10^{-4}$, respectively (Figure $2 \mathrm{C})$. The blue module contained a total of $66 \mathrm{mRNAs}$ and 22 lncRNAs, which were selected as malignant phenotypeassociated mRNAs and lncRNAs for the further analysis (Table 1).

\section{Identification of the malignant phenotype- associated miRNAs in HB}

We also identified tumor-associated miRNAs using WGCNA method based on GSE75283, which was miRNA profiling dataset derived from the same $\mathrm{HB}$ patients as GSE75271 (Figure 3A). Similarly, we divided 887 miRNAs into ten MEs, including MEgreen, MEpink, MEblack, MEtan, MEgreenyellow, MEmidnoghtblue, MEpurple, MEsalmon, MEblue and MEmagenta (Figure $3 \mathrm{~B})$. Interestingly, the module-trait relationship analysis revealed that MEmidnoghtblue was positively associated with HB subtypes, Cairo classification and tumor stage, with $P$-values of $2 \times 10^{-4}, 3 \times 10^{-4}$ and $2 \times 10^{-4}$, respectively (Figure 3C). The midnoghtblue module contained 13 miRNAs, which were identified as malignant phenotypeassociated miRNAs (Table 1).

\section{Coexpression analysis of malignant phenotype- associated molecules}

To explore the possible relationship between malignant phenotype-associated molecules, including 
mRNAs, lncRNAs and miRNAs, we performed coexpression analysis. As shown in Figure 4, we found that the lncRNAs were positively associated with nearly almost mRNAs. Interestingly, we also found that identified miRNAs were negatively associated with both mRNAs and lncRNAs. These results suggested that these mRNAs, lncRNAs and miRNAs might form lncRNAmiRNA-mRNA network, which affected the progression of HB patients. In order to predict biological function of above identified lncRNA-miRNA-mRNA network, we performed Gene ontology (GO) and Kyoto Encyclopedia of Genes and Genomes (KEGG) pathway enrichment analysis. Biological process analysis suggested these molecules were mainly involved in metabolic biological processes, such as lipoprotein metabolic process and oxidation-reduction process (Figure 5A). Cellular

A

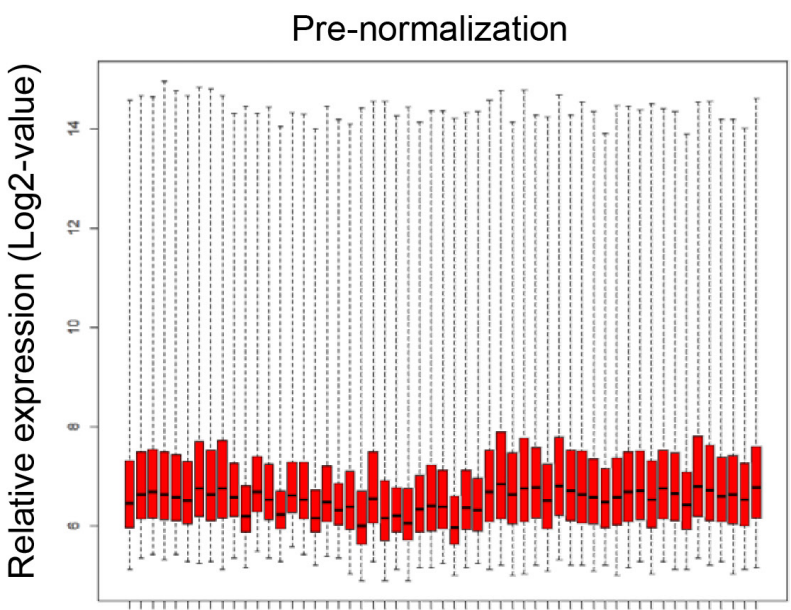

HB Samples

B

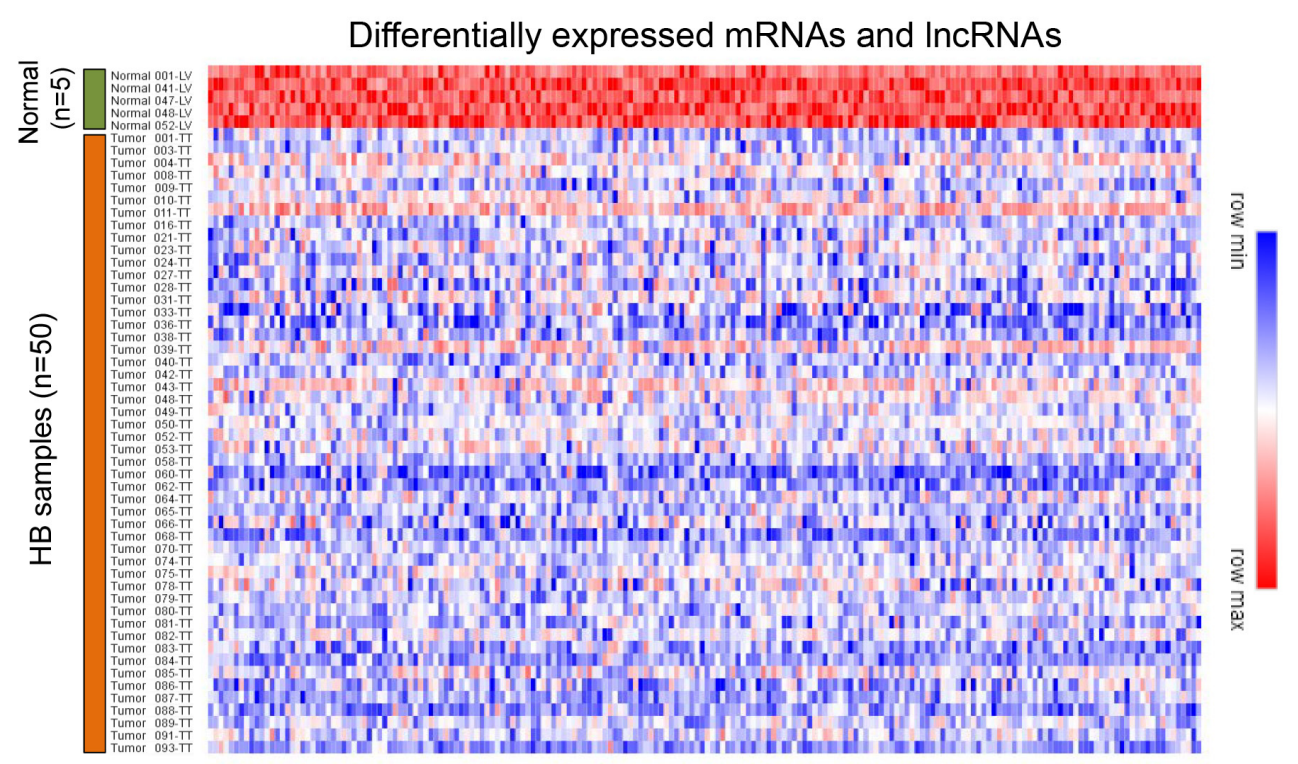

components analysis showed that they were located at endoplasmic reticulum (ER) membrane, the latter of which played a critical role in liver metabolism (Figure 5B). Besides, molecular function analysis also showed these molecules mostly belonged to heme-binding and oxygenbinding protein families (Figure 5C). KEGG pathway analysis further revealed that these malignant phenotypeassociated molecules might be involved in regulating the metabolic related pathways to influence carcinogenesis and tumor progression (Figure 5D).

\section{Construction of IncRNA-miRNA-mRNA network in $\mathrm{HB}$}

We further conducted IncRNA-miRNA-mRNA network in $\mathrm{HB}$ based on the coexpression analysis

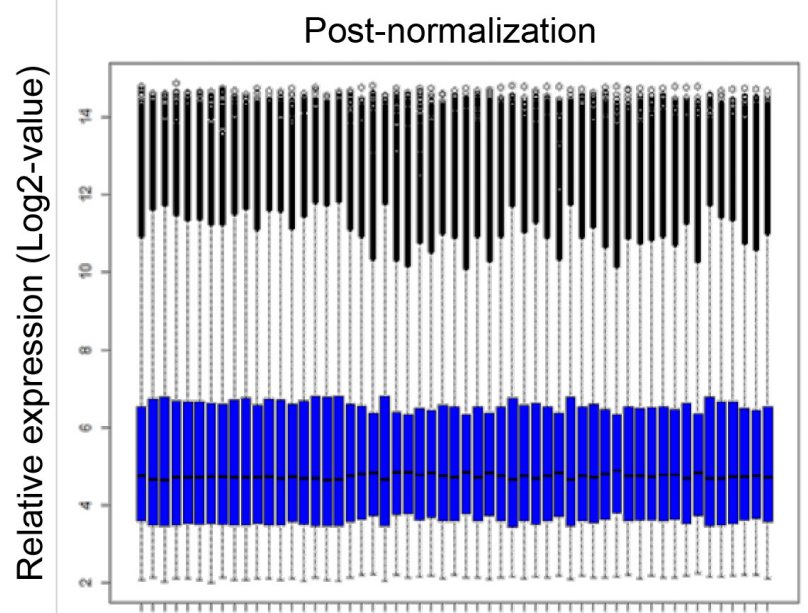

HB Samples

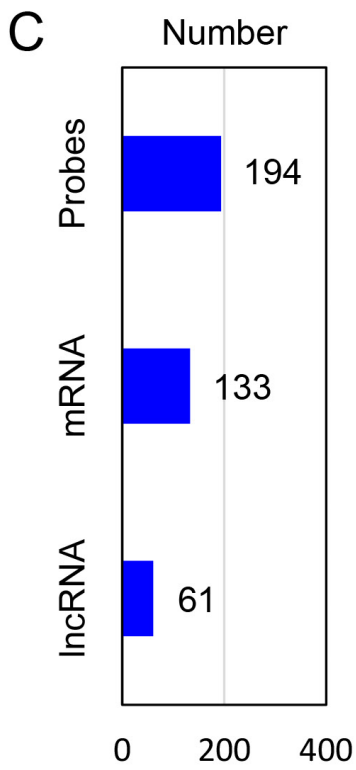

Figure 1: Identification of differentially expressed mRNA and IncRNAs from the HB dataset. (A) Normalization of dataset GSE75271. (B) A total of 194 downregulated probes were picked up from HB patients. (C) Within the 194 downregulated probes, 133 mRNAs and 61 lncRNAs were identified. 
of malignant phenotype-associated molecules. The construction of lncRNA-miRNA-mRNA network included three steps: (a) candidate malignant phenotype-associated lncRNAs, miRNAs and mRNAs were selected according to WGCNA results; (b) the correlation coefficients between IncRNAs, miRNAs and mRNAs were calculated based on their corresponding profiling data; (c) targets of miRNAs were predicted from Targetscan (http://www. targetscan.org/). Finally, a total of 6 miRNAs (hsa-miR106b, hsa-miR-130b, hsa-miR-19a, hsa-miR-19b, hsamiR-20a and hsa-miR-301a), 8 lncRNAs (NR 102317, XR_245338, XR_428373, XR_924945, XR_929728,
XR_931611, XR_935074 and XR_946696), and 6 mRNAs (EGFR, GAREM, INSIG1, KRT81, SAR1B and $S D C 1$ ) were selected to conduct lncRNA-miRNAmRNA network. Heatmap was conducted to present the correlation coeffecient between molecules (Figure 6A), and Cytoscape were used for visualization of lncRNAmiRNA-mRNA network (Figure 6B).

\section{DISCUSSION}

$\mathrm{HB}$ is a common malignant hepatic tumor in children and the prognosis varies among different

\section{B Clustering of module eigengenes}

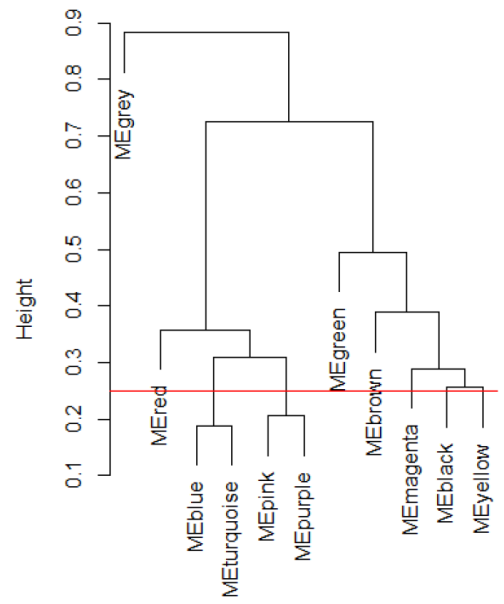

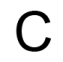

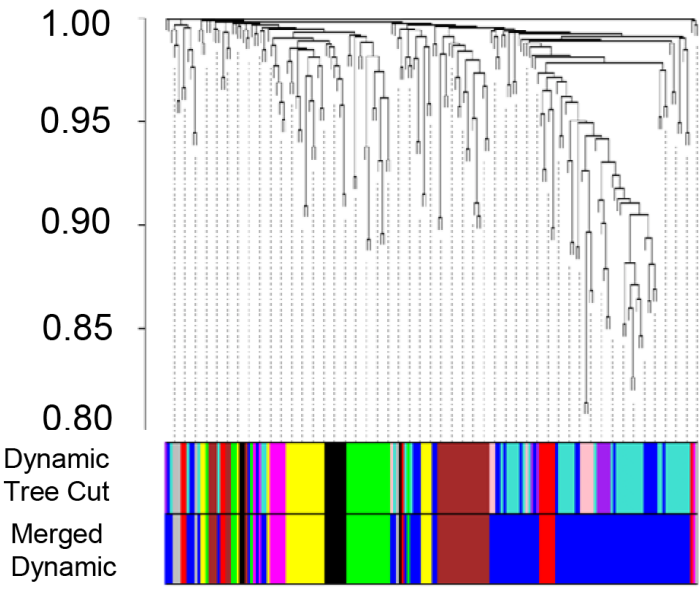

Module-trait relationships

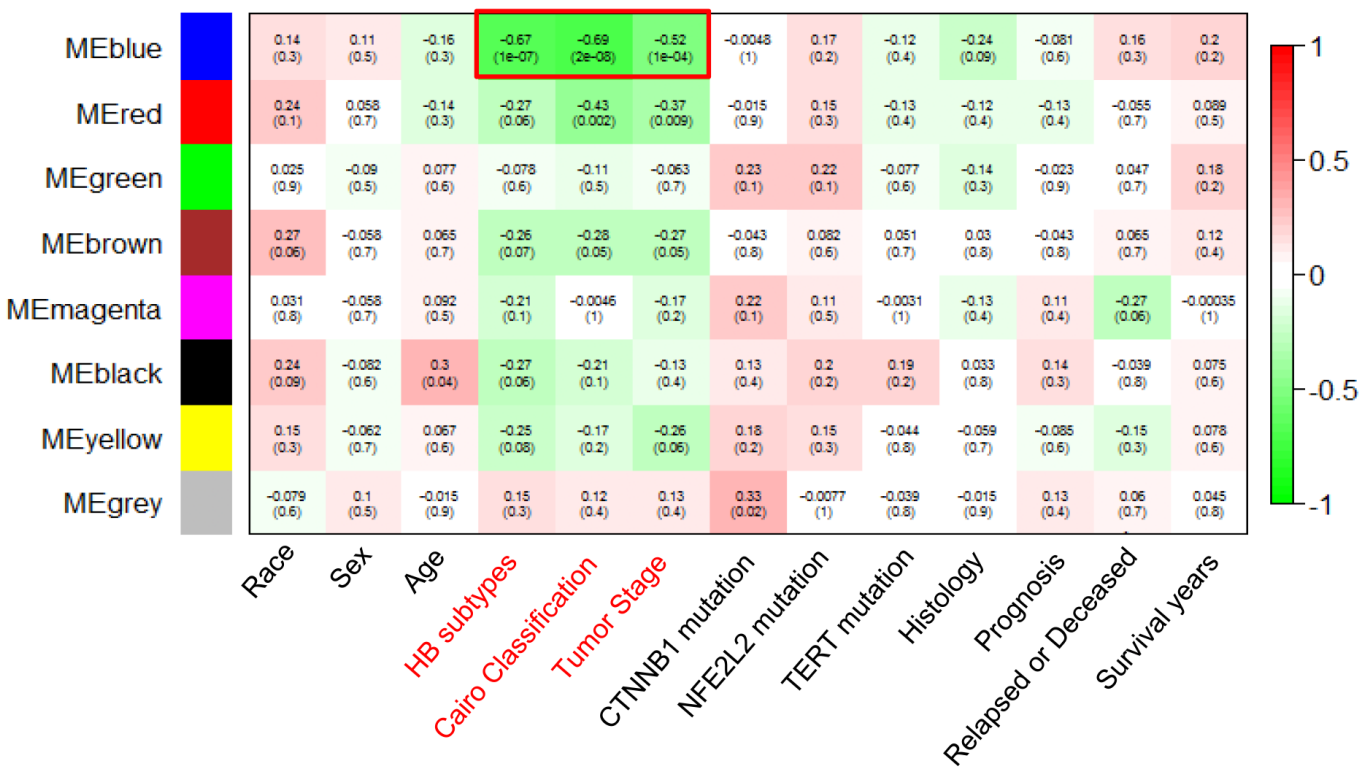

Figure 2: The WGCNA analysis of the malignant phenotype-associated mRNAs and IncRNAs. (A) The cluster dendrogram of differentially expressed mRNAs and lncRNAs derived from the dataset GSE75271. (B) The cluster dendrogram of module eigengenes. (C) The module-trait relationship analysis between the 8 odules and clinical characteristics. ME: module eigengenes. 
Table 1: Identification of HB-associated mRNAs, miRNAs and IncRNAs

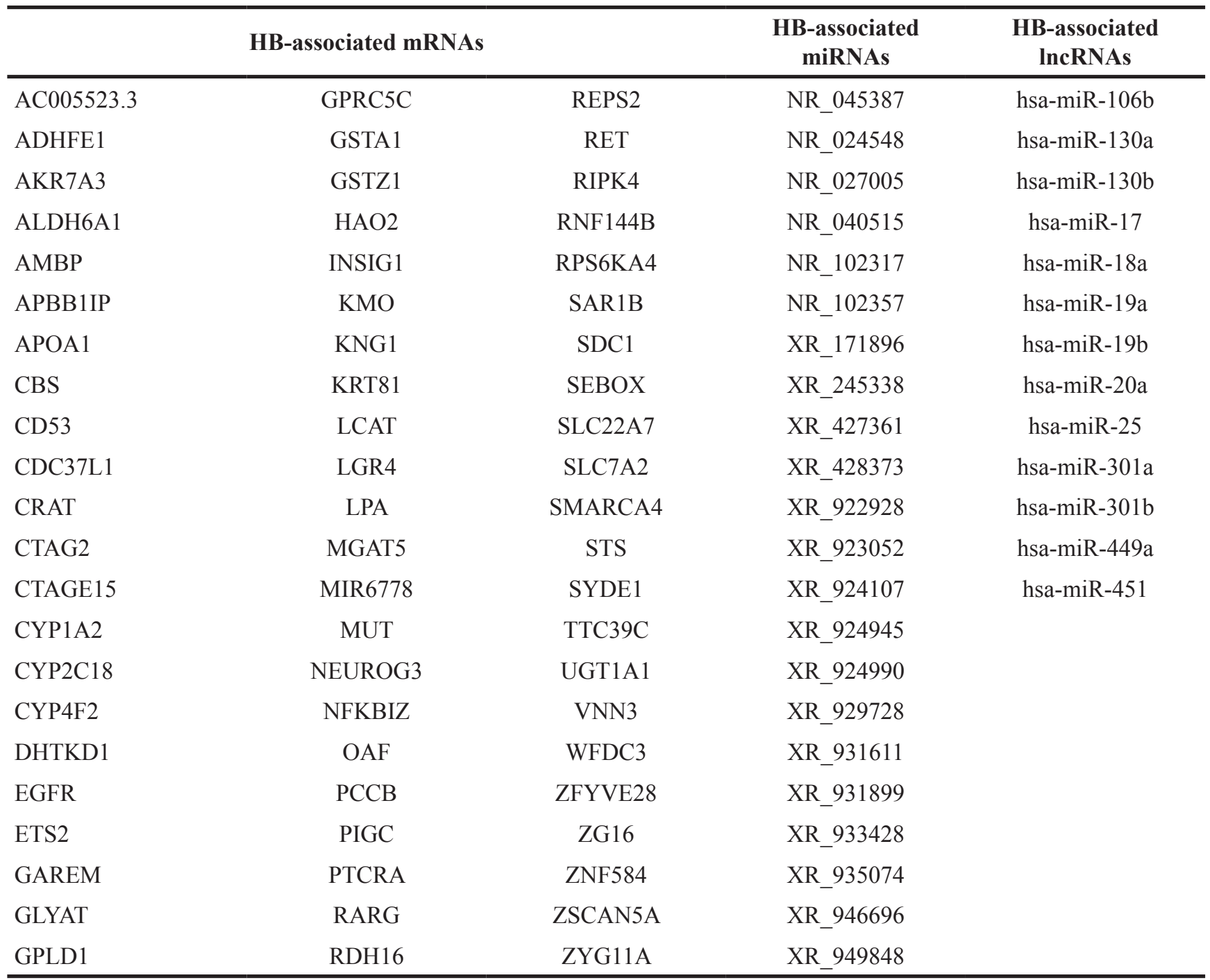

categories. To date, there are limited effective methods to treat HB. Hence, it's essential to explore underlying molecular mechanism of HB. In the present study, we for the first time identified the malignant phenotypeassociated lncRNAs, mRNAs and miRNAs, which were associated with HB subtypes, Cairo Classification and tumor stage. Next, we conducted the lncRNA-miRNAmRNA network based on their expression patterns to provide a molecular explanation for HB patients.

With the advancement of whole-genomic sequencing technologies, lncRNAs have attracted increasing attention [24]. Amount studies have reported that aberrant lncRNA expressions could serve as novel molecular biomarkers in the cancer diagnosis and prognosis prediction $[25,26]$. LncRNAs could function as oncogenes, regulating alternation of key signaling pathways, and promoting tumor growth and metastasis $[27,28]$. Recently, the miRNA sponge role of lncRNAs and IncRNA-miRNA-mRNA network have been widely accepted [20]. For instance, IncRNAs SPRY4-IT1 could sponge miR-101-3p and consequently increase EZH2 expression to promote cellular proliferation and metastasis of bladder cancer cells [29]. LncRNA-UCC was found to promote colorectal cancer progression by sponging miR143 [30], and lncRNA-CCAT1 promotes hepatocellular carcinoma progression by sponging let-7 [31]. However, whether the IncRNA-miRNA-mRNA network plays an important role in HB patients have not been fully explored. A recent study reported that TUG1/miR-34a-5p/VEGFA network was involved in regulating hypervascularity and hepatoblastoma progression [32]. In the present study, we, for the first time, explored malignant phenotype-associated lncRNAs, miRNAs and mRNAs in HB by using WGCNA methods, respectively. A total of 22 lncRNAs, 13 miRNAs and 66 mRNAs were identified, which were strongly associated with HB subtypes, Cairo classification and tumor stage. These findings may provide us potential biomarkers and/or anti-cancer targets in future. 
One hallmark of cancer is the metabolic reprogramming in cancer cells, including glycometabolism, lipid metabolism and amino acid metabolism [33-35]. In HB cell lines HepG2, Hep3B and $\mathrm{HuH}-6$, the metabolic reprogramming has been defined as one important hallmark and contributes to tumor progression [36]. Several driver genes of HB, such as $\beta$-catenin, YAP and c-Myc $[37,38]$, could also affect intracellular energy metabolism. Moreover, it was also be found that that molecular targeting of mitochondrial metabolism holds promise as a novel and effective therapeutic approach for HB [39]. In the present study, we also performed pathway enrichment analysis of above identified molecules. Our results revealed that most molecules associated with HB classification and tumor stage belonged to metabolic pathway, indicating a close interaction between metabolic pathways and HB malignant phenotypes.
A

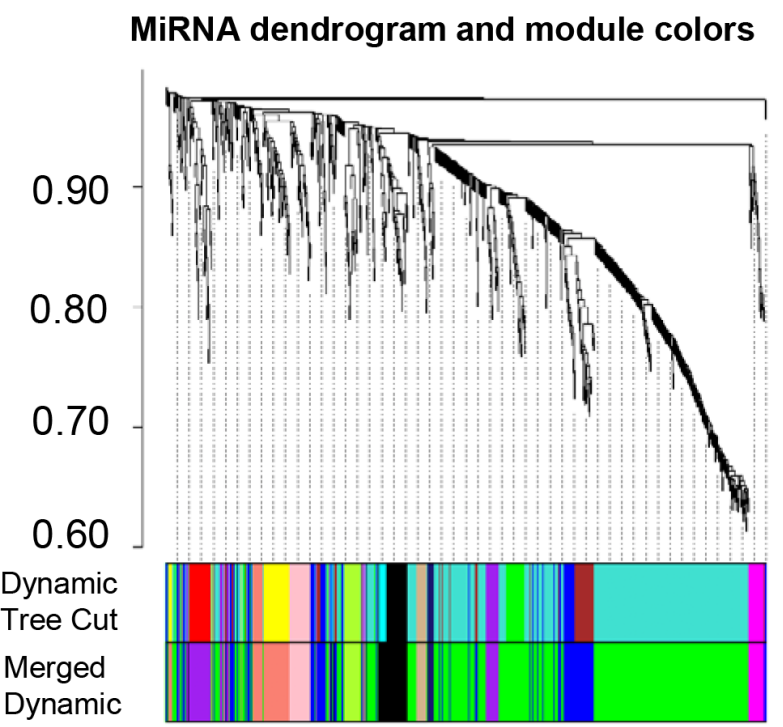

B Clustering of module eigengenes

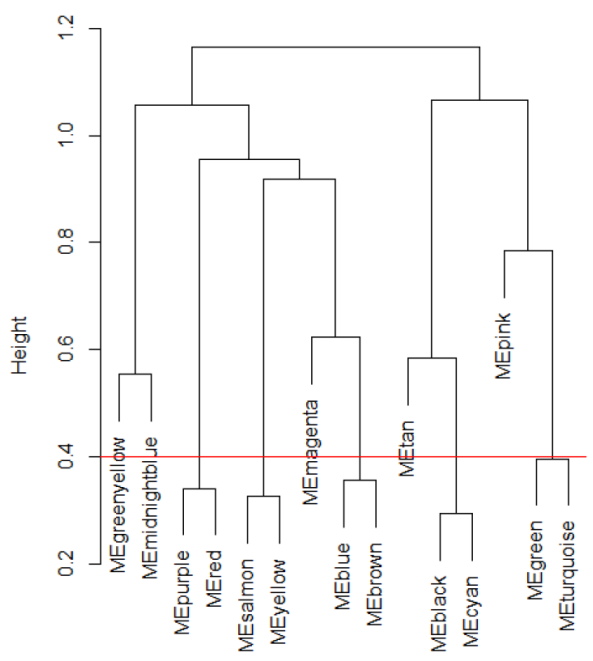

C

Module-trait relationships

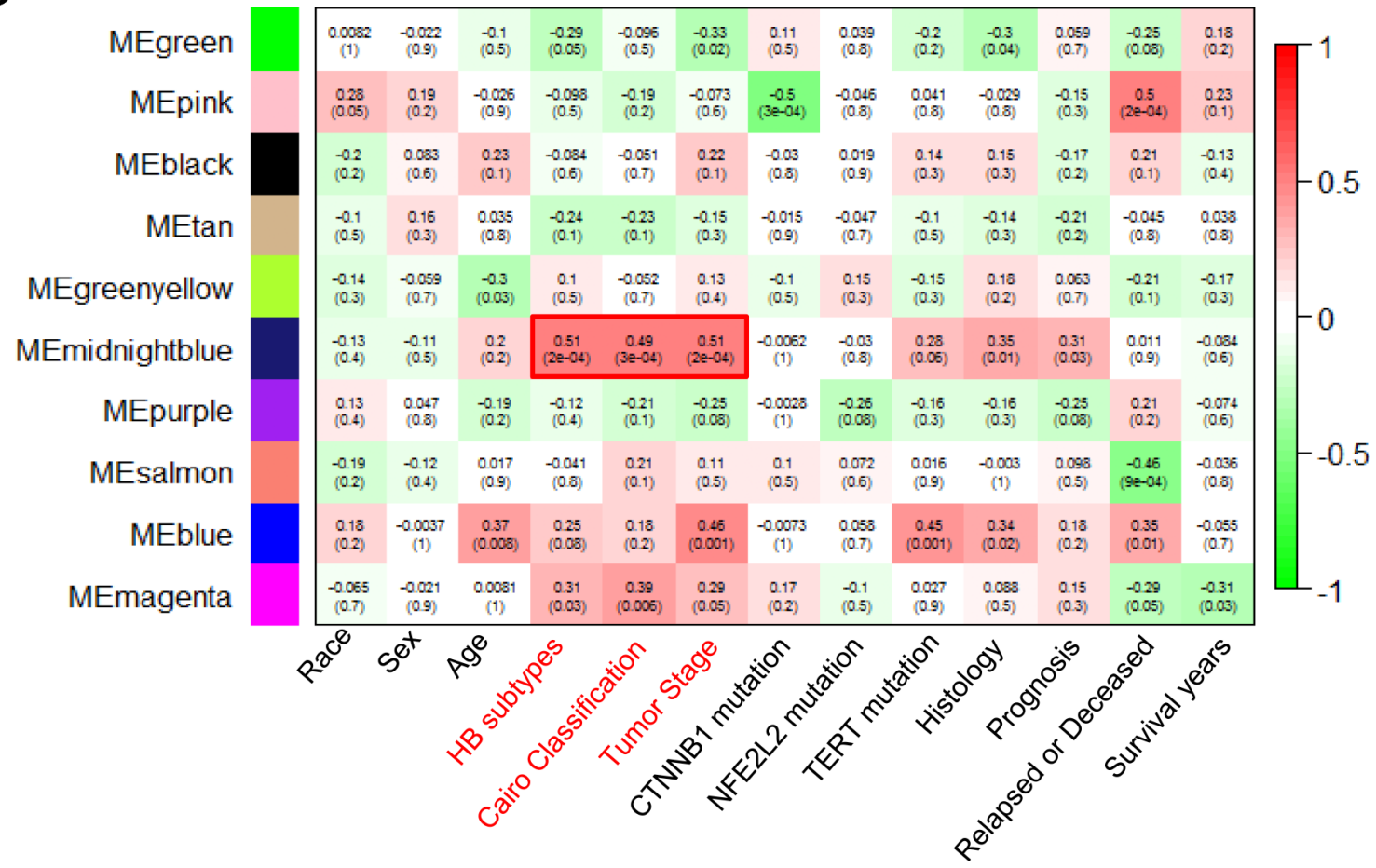

Figure 3: The WGCNA analysis of the malignant phenotype-associated miRNAs. (A) The cluster dendrogram of differentially expressed miRNAs derived from the dataset GSE75283. (B) The cluster dendrogram of module eigengenes. (C) The module-trait relationship analysis between the 10 modules and clinical characteristics. ME: module eigengenes. 
In this study, we also conducted the lncRNAmiRNA-mRNA network consisting of 6 miRNAs, 8 lncRNAs and 6 mRNAs, which was associated with HB classification and tumor stage. In this conducted network, we identified two genes in epidermal growth factor (EGF) signaling, EGFR and SDC1 [40], acting as effector genes. Recently, a whole transcriptome analysis based on HB patients suggested that aberrant EGF signaling was associated with HB classification [41]. The loss of EGFR signaling members were shown to be more present in less differentiated embryonal and undifferentiated small cells subtypes of HB. Therefore, our findings might also provide evidence for explaining the underlying molecular variation of different HB subtypes.

In conclusion, we identified malignant phenotypeassociated lncRNAs, miRNAs and mRNAs by employing WGCNA method. Mechanistically, these molecules might promote the malignant phenotypes of $\mathrm{HB}$ via regulating metabolic pathways. Moreover, we also conducted a lncRNAs-miRNAs-mRNAs network based on above findings. To our best knowledge, this is the first comprehensive study investigating lncRNA-miRNA-mRNA network in HB. If validated, our findings might provide evidence for exploring anti-cancer target in HB patients.

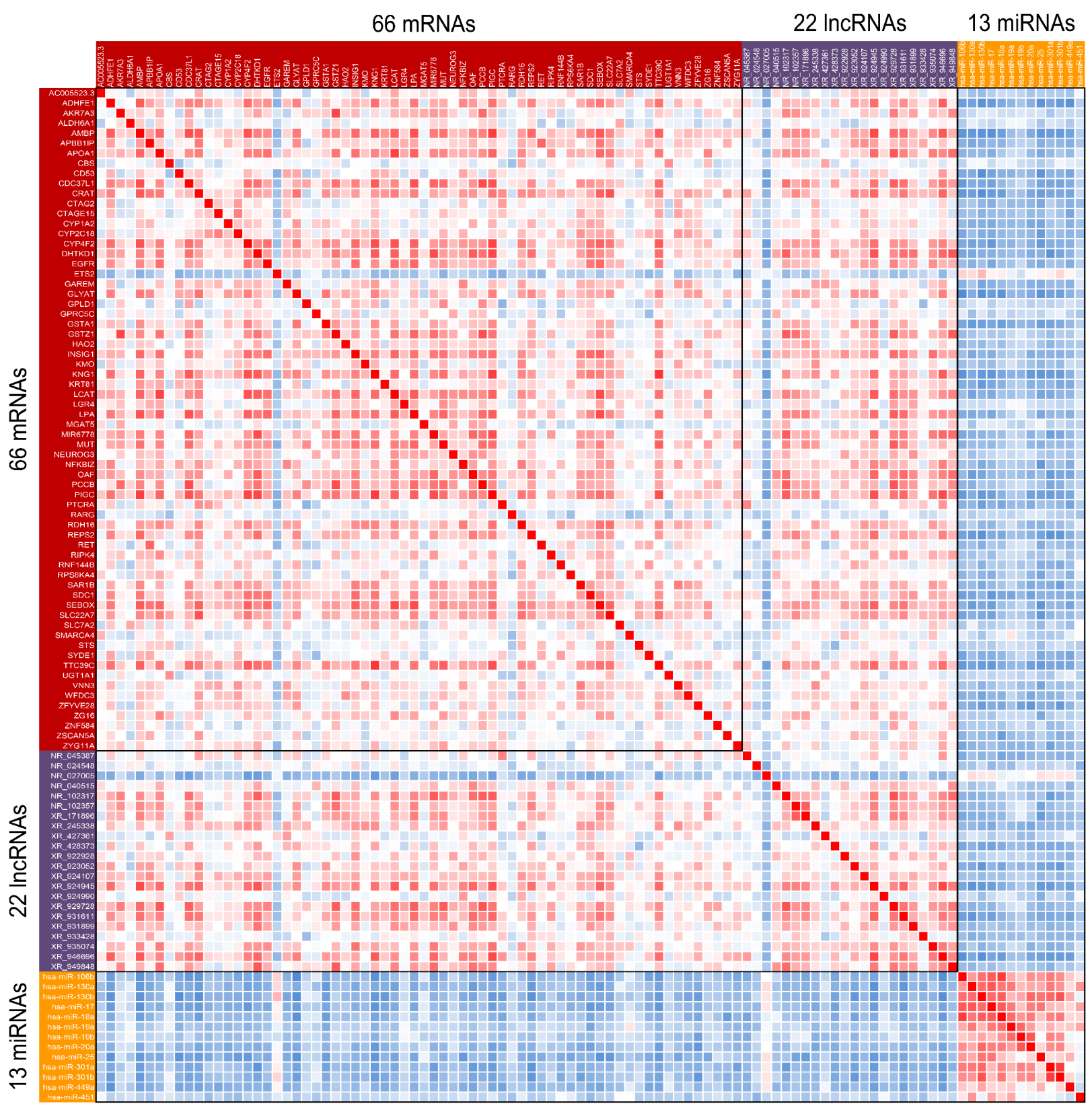

Figure 4: Coexpression analysis of malignant phenotype-associated molecules. The heatmap was presented by correlation coefficient between each pair of malignant phenotype-associated molecules. Range of colors (red to blue) shows the correlation coefficient (high to low). 
A

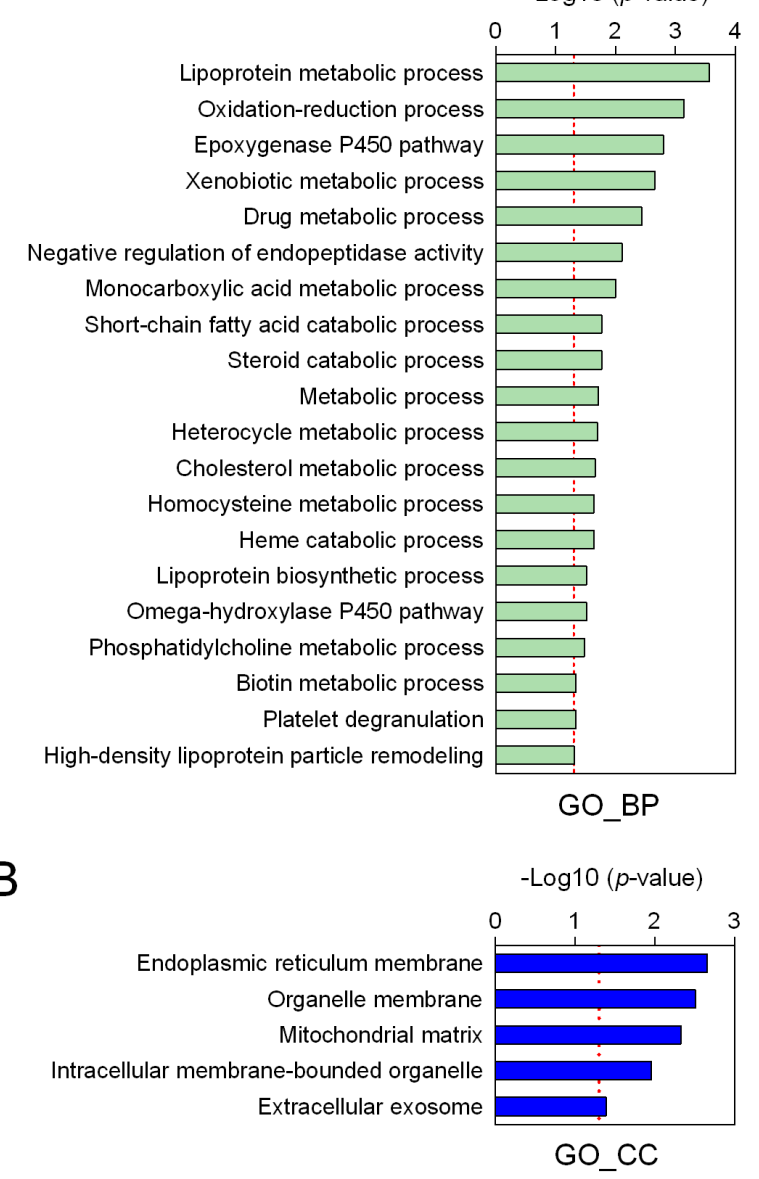

C
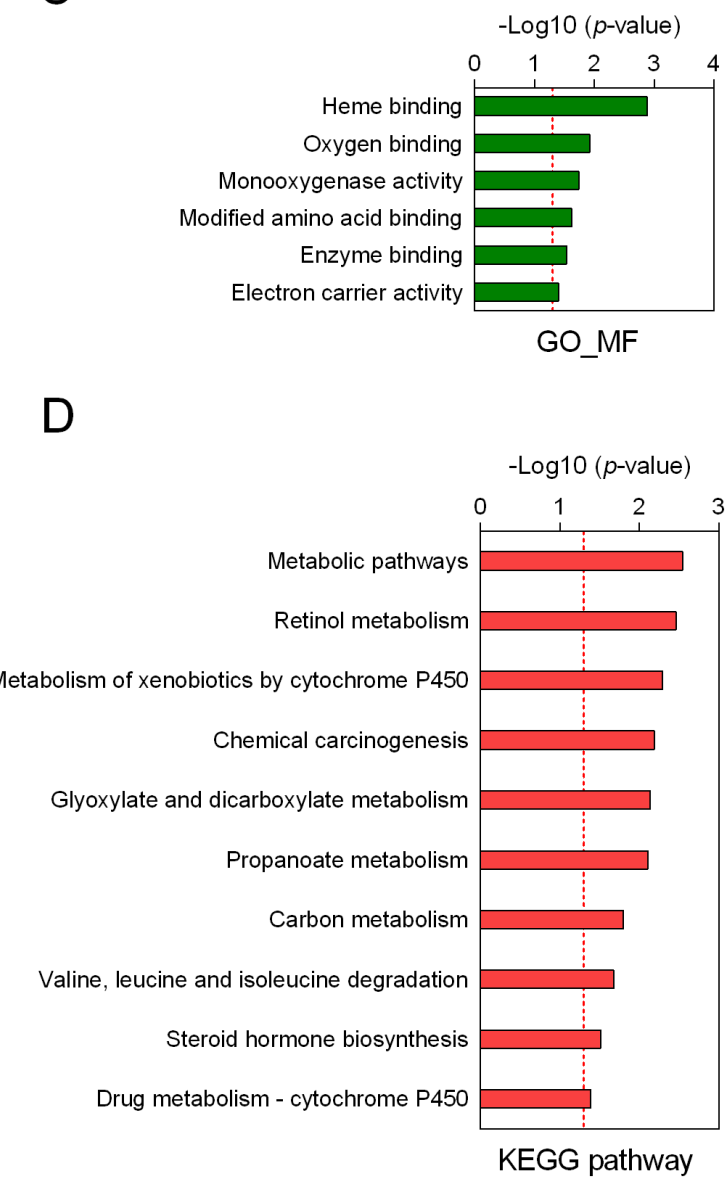

Figure 5: Function annotation of malignant phenotype-associated molecules. Gene ontology analysis of biological processes (A), cellular components (B) and molecular function (C) of those molecules. (D) The KEGG pathway enrichment analysis.

A

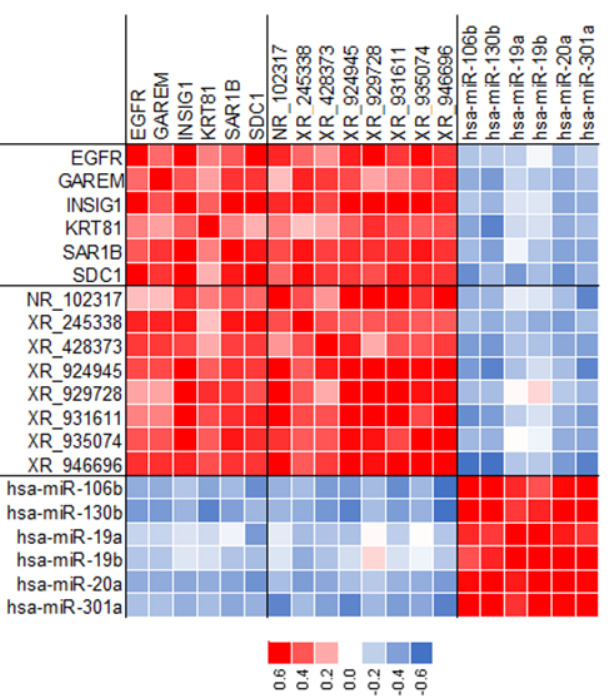

B

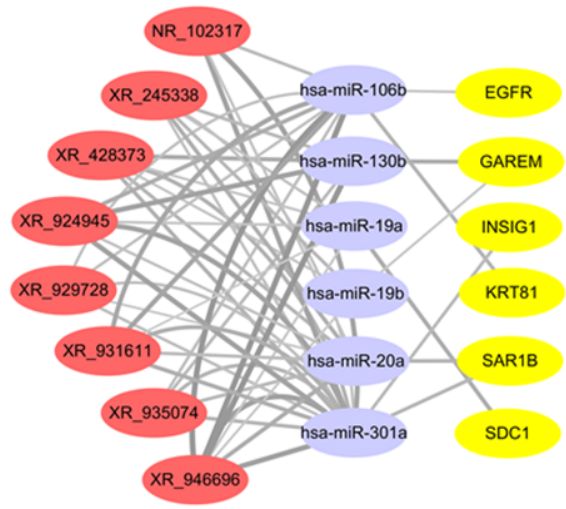

Figure 6: Construction of IncRNA-miRNA-mRNA network in HB. (A) Selected 6 mRNAs, 8 lncRNAs and 6 miRNAs for network. (B) Predicted lncRNA-miRNA-mRNA network in HB. 


\section{MATERIALS AND METHODS}

\section{Microarray data}

Gene and miRNA profiling data of HB patients were searched from the Gene Expression Omnibus (GEO, http://www.ncbi.nlm.nih.gov/geo), which is a public database containing freely available profiling datasets. We finally selected two datasets for bioinformatic analysis, including GSE75271 (mRNA profiling data based on Affymetrix Human Genome U133 Plus 2.0 Array platform) and GSE75283 (miRNA profiling data based on Agilent-029297 Human miRNA Microarray v15 platform), which were derived from the same $50 \mathrm{HB}$ patients and 5 controls [22]. All raw data were downloaded from GEO database.

\section{Data normalization and probe annotation}

The raw data of GSE75271 was stored as probelevel CEL files, and was quantile normalized using Robust Multi-array Average (RMA) method. After data normalization, expression level of each probe in GSE75271 was obtained. The probe sequences with corresponding Affymetrix probe IDs were downloaded from the Affymetrix website (http://www.affymetrix.com). The annotation of IncRNA transcripts were then performed according to previous reported method [23]. A total of 8240 lncRNA transcripts were generated with RefSeq transcript IDs. The probe ID-centric gene expression profile was also generated according to Affymetrix annotation files.

\section{Weighted gene correlation network analysis}

WGCNA is an algorithm for constructing a coexpression network, defined by the similarity of gene co-expression [42]. In data processing, the genome-wide gene expression data was initially filtrated with measuring the consistency of gene expression profiles by Pearson correlation, then we utilized the power adjacent function to Pearson correlation matrix to transform data into weighted gene co-expression networks. Network module represents a cluster of closely interconnected genes. Finally, the adjacency matrix, a measurement of topology similarity, is converted into the topological overlap matrix (TOM), and modules are detected by cluster analysis [43].

\section{Pathway enrichment analysis}

GO analysis is freely available for users in the annotation and biological properties of genes, gene products and sequences [44]. KEGG is a knowledge base for systematic analysis of gene functions in terms of the networks of genes and molecules [45]. We used GO analysis and KEGG analysis to identify the function of the aberrantly expressed candidate lncRNAs-associated genes.
The $P$-value of each enriched pathway was assigned with $-\log 10$ transformation. $P<0.05(-\log 10(P$-value $)=1.30)$ was considered as statistically significant.

\section{Statistical analysis}

All data were analyzed by R software 3.4.1 (https:// www.r-project.org/). For the pair of molecule $i$ and $j$, Pearson correlation coefficient was computed. $P<0.05$ was considered statistically significant.

\section{Abbreviations}

ceRNAs: competing endogenous RNAs; ER: endoplasmic reticulum; EGF: epidermal growth factor; GEO: Gene Expression Omnibus; GO: Gene ontology; HB: Hepatoblastoma; KEGG: Kyoto Encyclopedia of Genes and Genomes; LIMMA: linear models for microarray data; lncRNA: long non-coding RNAs; miRNA: microRNAs; MRE: miRNA response elements; ME: module eigengenes; RMA: Robust Multi-array Average; TOM: topological overlap matrix; WGCNA: weighted gene correlation network analysis.

\section{Author contributions}

Kai Qu and Shunbin Dong: Designed the research and analyzed the data; Sida Liu and Fujing Xie: Collected and analyzed data; Sinan Liu: Constructed figures; Xiaohong Xiang and Kai Qu: Drafted and revised the manuscript.

\section{CONFLICTS OF INTEREST}

The authors declare no conflicts of interest.

\section{FUNDING}

This work was supported by National Science Foundation of China (No. 81201549); Natural Science Basic Research Plan in Shaanxi Province of China (No. 2017JM8039); Fundamental Research Fund for the Central Universities (No. 2016qngz05); the Clinical Research Award of the First Affiliated Hospital of Xi'an Jiaotong University, China (No. XJTU1AF-CRF-2015-011).

\section{REFERENCES}

1. Bosman F, Carneiro F, Hruban R, Theise N. World Health Organization Classification of Tumours of the Digestive System. 2012.

2. Spector LG, Birch J. The epidemiology of hepatoblastoma. Pediatr Blood Cancer. 2012; 59:776-779.

3. Dong R, Jia D, Xue P, Cui X, Li K, Zheng S, He X, Dong K. Genome-wide analysis of long noncoding RNA (lncRNA) 
expression in hepatoblastoma tissues. PLoS One. 2014; 9:e85599.

4. Rowland JM. Hepatoblastoma: assessment of criteria for histologic classification. Med Pediatr Oncol. 2002; 39:478-483.

5. Stocker JT. Hepatoblastoma. Semin Diagn Pathol. 1994; 11:136-143.

6. Kremer N, Walther AE, Tiao GM. Management of hepatoblastoma: an update. Curr Opin Pediatr. 2014; 26:362-369.

7. Czauderna P, Lopez-Terrada D, Hiyama E, Haberle B, Malogolowkin MH, Meyers RL. Hepatoblastoma state of the art: pathology, genetics, risk stratification, and chemotherapy. Curr Opin Pediatr. 2014; 26:19-28.

8. McCarville MB, Roebuck DJ. Diagnosis and staging of hepatoblastoma: imaging aspects. Pediatr Blood Cancer. 2012; 59:793-799.

9. Malogolowkin MH, Katzenstein HM, Meyers RL, Krailo MD, Rowland JM, Haas J, Finegold MJ. Complete surgical resection is curative for children with hepatoblastoma with pure fetal histology: a report from the Children's Oncology Group. J Clin Oncol. 2011; 29:3301-3306.

10. Maibach R, Roebuck D, Brugieres L, Capra M, Brock P, Dall'Igna P, Otte JB, De Camargo B, Zsiros J, Zimmermann A, Aronson D, Childs M, Scopinaro M, et al. Prognostic stratification for children with hepatoblastoma: the SIOPEL experience. Eur J Cancer. 2012; 48:1543-1549.

11. Aronson DC, Schnater JM, Staalman CR, Weverling GJ, Plaschkes J, Perilongo G, Brown J, Phillips A, Otte JB, Czauderna P, MacKinlay G, Vos A. Predictive value of the pretreatment extent of disease system in hepatoblastoma: results from the International Society of Pediatric Oncology Liver Tumor Study Group SIOPEL-1 study. J Clin Oncol. 2005; 23:1245-1252.

12. Consortium EP. An integrated encyclopedia of DNA elements in the human genome. Nature. 2012; 489:57-74

13. Carninci P, Kasukawa T, Katayama S, Gough J, Frith MC, Maeda N, Oyama R, Ravasi T, Lenhard B, Wells C, Kodzius R, Shimokawa K, Bajic VB, et al. The transcriptional landscape of the mammalian genome. Science. 2005; 309:1559-1563.

14. Lander ES, Linton LM, Birren B, Nusbaum C, Zody MC, Baldwin J, Devon K, Dewar K, Doyle M, FitzHugh W, Funke R, Gage D, Harris K, et al. Initial sequencing and analysis of the human genome. Nature. 2001; 409:860-921.

15. Ambros V. The functions of animal microRNAs. Nature. 2004; 431:350-355.

16. Iyer MK, Niknafs YS, Malik R, Singhal U, Sahu A, Hosono Y, Barrette TR, Prensner JR, Evans JR, Zhao S, Poliakov A, Cao X, Dhanasekaran SM, et al. The landscape of long noncoding RNAs in the human transcriptome. Nat Genet. 2015; 47:199-208.

17. Khalil AM, Guttman M, Huarte M, Garber M, Raj A, Rivea Morales D, Thomas K, Presser A, Bernstein BE, van Oudenaarden A, Regev A, Lander ES, Rinn JL. Many human large intergenic noncoding RNAs associate with chromatin-modifying complexes and affect gene expression. Proc Natl Acad Sci U S A. 2009; 106:11667-11672.

18. Rinn JL, Chang HY. Genome regulation by long noncoding RNAs. Annu Rev Biochem. 2012; 81:145-166.

19. Spitale RC, Tsai MC, Chang HY. RNA templating the epigenome: long noncoding RNAs as molecular scaffolds. Epigenetics. 2011; 6:539-543.

20. Salmena L, Poliseno L, Tay Y, Kats L, Pandolfi PP. A ceRNA hypothesis: the Rosetta Stone of a hidden RNA language? Cell. 2011; 146:353-358.

21. Arunkumar G, Murugan AK, Prasanna Srinivasa Rao $\mathrm{H}$, Subbiah S, Rajaraman R, Munirajan AK. Long noncoding RNA CCAT1 is overexpressed in oral squamous cell carcinomas and predicts poor prognosis. Biomed Rep. 2017; 6:455-462.

22. Sumazin P, Chen Y, Trevino LR, Sarabia SF, Hampton OA, Patel K, Mistretta TA, Zorman B, Thompson P, Heczey A, Comerford S, Wheeler DA, Chintagumpala M, et al. Genomic analysis of hepatoblastoma identifies distinct molecular and prognostic subgroups. Hepatology. 2017; 65:104-121.

23. Zhang X, Sun S, Pu JK, Tsang AC, Lee D, Man VO, Lui WM, Wong ST, Leung GK. Long non-coding RNA expression profiles predict clinical phenotypes in glioma. Neurobiol Dis. 2012; 48:1-8.

24. Wang KC, Chang HY. Molecular mechanisms of long noncoding RNAs. Mol Cell. 2011; 43:904-914.

25. Meng J, Li P, Zhang Q, Yang Z, Fu S. A four-long noncoding RNA signature in predicting breast cancer survival. J Exp Clin Cancer Res. 2014; 33:84.

26. Hu Y, Chen HY, Yu CY, Xu J, Wang JL, Qian J, Zhang X, Fang JY. A long non-coding RNA signature to improve prognosis prediction of colorectal cancer. Oncotarget. 2014; 5:2230-2242. https://doi.org/10.18632/oncotarget.1895.

27. Qiu MT, Hu JW, Yin R, Xu L. Long noncoding RNA: an emerging paradigm of cancer research. Tumour Biol. 2013; 34:613-620.

28. Shi X, Sun M, Liu H, Yao Y, Song Y. Long non-coding RNAs: a new frontier in the study of human diseases. Cancer Lett. 2013; 339:159-166.

29. Liu D, Li Y, Luo G, Xiao X, Tao D, Wu X, Wang M, Huang C, Wang L, Zeng F, Jiang G. LncRNA SPRY4-IT1 sponges miR-101-3p to promote proliferation and metastasis of bladder cancer cells through up-regulating EZH2. Cancer Lett. 2017; 388:281-291.

30. Huang FT, Chen WY, Gu ZQ, Zhuang YY, Li CQ, Wang LY, Peng JF, Zhu Z, Luo X, Li YH, Yao HR, Zhang SN. The novel long intergenic noncoding RNA UCC promotes colorectal cancer progression by sponging miR-143. Cell Death Dis. 2017; 8:e2778.

31. Deng L, Yang SB, Xu FF, Zhang JH. Long noncoding RNA CCAT1 promotes hepatocellular carcinoma progression by 
functioning as let-7 sponge. J Exp Clin Cancer Res. 2015; $34: 18$.

32. Dong R, Liu GB, Liu BH, Chen G, Li K, Zheng S, Dong KR. Targeting long non-coding RNA-TUG1 inhibits tumor growth and angiogenesis in hepatoblastoma. Cell Death Dis. 2016; 7:e2278.

33. Ward PS, Thompson CB. Metabolic reprogramming: a cancer hallmark even warburg did not anticipate. Cancer Cell. 2012; 21:297-308.

34. Currie E, Schulze A, Zechner R, Walther TC, Farese RV Jr. Cellular fatty acid metabolism and cancer. Cell Metab. 2013; 18:153-161.

35. Zhang F, Du G. Dysregulated lipid metabolism in cancer. World J Biol Chem. 2012; 3:167-174.

36. Byun JK, Choi YK, Kang YN, Jang BK, Kang KJ, Jeon YH, Lee HW, Jeon JH, Koo SH, Jeong WI, Harris RA, Lee IK, Park KG. Retinoic acid-related orphan receptor alpha reprograms glucose metabolism in glutamine-deficient hepatoma cells. Hepatology. 2015; 61:953-964.

37. Wang H, Lu J, Edmunds LR, Kulkarni S, Dolezal J, Tao J, Ranganathan S, Jackson L, Fromherz M, Beer-Stolz D, Uppala R, Bharathi S, Monga SP, et al. Coordinated activities of multiple Myc-dependent and Myc-independent biosynthetic pathways in hepatoblastoma. J Biol Chem. 2016; 291:26241-26251.

38. Zheng K, Cubero FJ, Nevzorova YA. c-MYC-making liver sick: role of c-MYC in hepatic cell function, homeostasis and disease. Genes (Basel). 2017; 8:123.
39. Glushakova LG, Lisankie MJ, Eruslanov EB, OjanoDirain C, Zolotukhin I, Liu C, Srivastava A, Stacpoole PW. AAV3-mediated transfer and expression of the pyruvate dehydrogenase E1 alpha subunit gene causes metabolic remodeling and apoptosis of human liver cancer cells. Mol Genet Metab. 2009; 98:289-299.

40. Wang H, Jin H, Rapraeger AC. Syndecan-1 and Syndecan-4 capture epidermal growth factor receptor family members and the alpha3betal integrin via binding sites in their ectodomains: novel synstatins prevent kinase capture and inhibit alpha6beta4-integrin-dependent epithelial cell motility. J Biol Chem. 2015; 290:26103-26113.

41. Ranganathan S, Ningappa M, Ashokkumar C, Higgs BW, Min J, Sun Q, Schmitt L, Subramaniam S, Hakonarson H, Sindhi R. Loss of EGFR-ASAP1 signaling in metastatic and unresectable hepatoblastoma. Sci Rep. 2016; 6:38347.

42. Chen J, Yu L, Zhang S, Chen X. Network analysis-based approach for exploring the potential diagnostic biomarkers of acute myocardial infarction. Front Physiol. 2016; 7:615.

43. Zhao H, Cai W, Su S, Zhi D, Lu J, Liu S. Screening genes crucial for pediatric pilocytic astrocytoma using weighted gene coexpression network analysis combined with methylation data analysis. Cancer Gene Ther. 2014; 21:448-455.

44. Gene Ontology Consortium. The Gene Ontology (GO) project in 2006. Nucleic Acids Res. 2006; 34:D322-326.

45. Ogata H, Goto S, Sato K, Fujibuchi W, Bono H, Kanehisa M. KEGG: Kyoto encyclopedia of genes and genomes. Nucleic Acids Res. 1999; 27:29-34. 